\title{
Unified performance bounds for generalized selection diversity combining in fading channels
}

\author{
C. Tellambura \\ Dept. of Electrical and \\ Computer Engineering \\ University of Alberta \\ Edmonton, AB T6G 2V4, Canada \\ Telephone: (780) 492-7228 \\ Fax: (780) 492-1811 \\ Email: chintha@ee.ualberta.ca
}

\author{
A. Annamalai \\ Bradley Dept. of Electrical and \\ Computer Engineering \\ Virginia Tech \\ Blacksburg, VA 24061 USA \\ Telephone: (540) 231-2129 \\ Fax: (540) 231-2968 \\ email: annamalai@vt.edu
}

\begin{abstract}
A generalized selection combiner (GSC) ranks the $L$ available diversity paths and then combines a subset of $M$ paths with the highest signal-to-noise ratios (SNRs). The cumulative distribution function (cdf) and moment generating function (mgf) of a linear sum of ordered random variables are therefore needed for computing the outage probability and error rates of a GSC receiver. Unfortunately, except for simple cases, these functions are either not known in closed form or exist only as multidimensional integrals. Consequently, an exact performance analysis is extremely difficult and time-consuming for many fading environments (including mixed-fading case) specifically when $L$ is large. This paper therefore derives new upper and lower bounds for the cdf of GSC output SNR over generalized fading channels. These bounds are either derived in closed-form or as single integral expressions. These bound are then used to derive new bounds for the average symbol error rates for a multitude of binary and $M$-ary digital modulation schemes in a variety of fading channel models that heretofore had resisted simple solutions.
\end{abstract}

\section{INTRODUCTION}

The GSC diversity scheme has received much attention recently (see [1]-[9] among many others). The ability to capture significant amount of transmitted signal energy present in the resolvable multi-paths using only few rake fingers (correlators) is an important receiver design consideration for wideband CDMA and ultra-wideband communication systems. Even though the MRC-rake receiver solution (i.e., combining all the $L$ resolvable multipaths using maximal-ratio combining technique) is optimum, it may not be desirable for practical implementations for several reasons. For instance, MRC receiver complexity is dependent on the physical channel characteristics (i.e., the channel length $L$ may vary with operating environment as well as time), and therefore undesirable. When additional factors such as channel estimation errors and complexity-performance trade-off for MRC implementation with a large number of multipaths are taken into account, it is reasonable to combine only a few strongest multipaths. This fact motivates the development of suboptimal coherent generalized selection combining $(\operatorname{GSC}(N, L))$ technique [4] in which a subset of $N \leq L$ paths with highest signal-to-noise ratios (SNRs) are optimally weighted and summed. A GSC receiver can be analyzed in terms of order statistics. Suppose $\gamma_{k}(1 \leq k \leq L)$ are random variables (RVs) representing the SNRs of $L$ diversity branches. If the RVs are rearranged in ascending order and then written as $\gamma_{(1)} \leq \gamma_{(2)} \leq \cdots \leq \gamma_{(L)}$, we shall denote a receiver $\operatorname{GSC}(M, L)$ (where $1 \leq M \leq L$ ) as a linear combiner operating on the $M$ best possible paths (ie $\left.\gamma_{(L-M+1)}, \ldots, \gamma_{(L)}\right)$. Clearly, $\operatorname{GSC}(1, L)$ and $\operatorname{GSC}(L, L)$ are simply the well-known selection combining (SC) and classical maximal-ratio combining (MRC) receivers. With the emergence of ultra wideband (UWB) systems and other high capacity systems, there is a need for system designers to evaluate the performance of GSC receivers for large $L$ (say 20 or more) over a wide variety of fading models.

In this paper, we derive new upper and lower bounds for the outage and average error probability for GSC receivers. Interestingly, these bounds are not based upon the conventional Chernoff bound approach. Several factors motivate the derivation of bounds. Despite many recent papers on performance analysis for GSC receivers, simple analytical results exist only when the diversity branches experience independent and identically distributed (iid) Rayleigh-fading (for example, outage expressions have been published for this case only [10]). The mgf of the GSC output can be used to obtain the average error rates. The outage also in principle can be obtained from the mgf. A simple closed-form expression for the mgf exists only for the iid Rayleigh case ${ }^{1}$. Even for independent, non identically distributed (ind) Rayleigh fading statistics, the mgf expression is rather complex [10]. Although for iid Nakagami$\mathrm{m}$ statistics (where $m$ is limited to a positive integers), a closed-form mgf expression can be derived [11], it is again rather complex. A novel technique derived in [12], [13] allows the mgf to be expressed as a single integral for any common fading models such as Rice, Weibull and others. However, the outage which is in fact the cumulative density function (cdf) can not readily be obtained analytically as a Laplace inversion

\footnotetext{
${ }^{1}$ That is, the branch SNRs are iid exponential sample. The general problem of the mgf for any linear sum of ordered statistics remains an open problem even within the wider mathematical statistics community, where order statistics have been researched since 1930s.
} 
of the mgf and numerical Laplace inversion essentially leads to two-dimensional numerical integration. Since the Chernoff bounding approach requires the mgf, it also fails to be of use for a unified analysis. Our new outage bounds overcome these difficulties in that they can be applied to any fading model and can handle both iid and ind statistics. As well, it is extremely time-consuming to evaluate performance of GSC systems for a large number for multipath components (say in the order of 20 or more) and for ind fading environments such as in ultra wideband communications. This problem can be circumvented by our approach.

The paper is organized as follows. Section II summarizes both pdf $f_{\gamma}(x)$ and cdf $F_{\gamma}(x)$ of the received SNR per branch for several multipath fading distributions. Section III develops a set of simple bounds for the outage. Section IV develops a set of improved bounds for the outage. Section V develops average error rate bounds from the outage bounds. Section VI provides numerical results and concludes the paper.

We will adopt the following notational conventions. For any random variable $\xi$, the pdf, cdf and mean are denoted by $f_{\xi}(x), F_{\xi}(x)$ and $\bar{\xi}$ respectively. For ind multichannel reception, $f_{\gamma, i}(x)$ and $F_{\gamma, i}(x)$ denote the pdf and cdf of the $i$-th branch SNR, with the parameters of the fading model being denoted as $\bar{\gamma}_{i}, m_{i}$ and so on. We assume $L$ diversity branches available for processing and they are independently distributed (uncorrelated). A generalized fading channel implies two cases: $f_{\gamma, i}(x)$ 's all emanate from the same $f_{\gamma}(x)$ but have different average powers (ie, $\bar{\gamma}_{i}$ varies with $i$ ), or more generally $f_{\gamma, i}(x)$ 's come from different distribution families (mixed fading).

\section{FADING MODELS}

\section{A. Rayleigh Fading}

Fading signal amplitudes in large cells (macro-cellular environments) in the absence of a direct line of sight component or tropospheric and ionospheric propagation based on reflection and refraction are usually modeled as Rayleigh random variables. In macro-cellular environments, fading appears as Rayleigh distributed, because the transmitted field is heavily scattered between the transmitter and the receiver. The pdf and cdf of the instantaneous branch SNR are given by

$$
\begin{aligned}
& f_{\gamma}(x)=\frac{1}{\bar{\gamma}} \exp \left(-\frac{x}{\bar{\gamma}}\right), \quad x \geq 0 \\
& F_{\gamma}(x)=1-\exp \left(-\frac{x}{\bar{\gamma}}\right), \quad x \geq 0
\end{aligned}
$$

where $\bar{\gamma}=E[x]$ denotes the average SNR/symbol/branch.

\section{B. Rician Fading}

In micro-cellular (e.g., urban and suburban land mobile radio communications) and pico-cellular (local area networks and indoor communications) environments, there usually exist a dominant line of sight path in addition to numerous diffused multipath components between the transmitter and receiver. Line-of-sight or main paths are more likely to exist in such cells because cell size is smaller and the user is nearer to base stations. Thus, indoor fading is often expected to be Rice distributed. Experimental results at several frequencies support the Rician model. Fading signal amplitudes in this case follow the Rician distribution with the ratio between the specular and diffused components denoted by the Rice factor $K$. The pdf of instantaneous branch SNR (a noncentral chisquare distribution) and the corresponding cdf is given by

$$
\begin{aligned}
f_{\gamma}(x) & =\frac{1+K}{\bar{\gamma}} \exp \left[-K-\frac{(1+K) x}{\bar{\gamma}}\right] \\
& \times I_{0}\left[2 \sqrt{\frac{K(K+1) x}{\bar{\gamma}}}\right], x \geq 0 \\
F_{\gamma}(x) & =1-Q\left[\sqrt{2 K}, \sqrt{\frac{2(K+1) x}{\bar{\gamma}}}\right], x \geq 0
\end{aligned}
$$

where $Q(\sqrt{2 a}, \sqrt{2 b})=\int_{b}^{\infty} \exp (-t-a) I_{0}(2 \sqrt{a t}) d t$ is the first order Marcum Q-function, $I_{0}($.$) is the modified Bessel$ function of the first kind and $K \geq 0$ is the Rice factor. For the limiting case of $K=0$, the Rician distribution reduces to the Rayleigh distribution.

\section{Nakagami-m Fading}

The Nakagami- $m$ distribution with fading severity index $m \geq 1 / 2$ is a versatile statistical model as it models signals that experience either less or more severe fading that that of Rayleigh fading. It sometimes fits experimental data (channel measurements in urban and indoor propagation environments) much better than Rayleigh or Rician distributions [14], [15]. The model also includes the one-sided Gaussian $(m=1 / 2)$ and Rayleigh $(m=1)$ distributions as special instances, and closely approximates the Rician distribution via relationship $m=(K+1)^{2} /(2 K+1)$ [16]. The instantaneous branch SNR is a Gamma variate and its pdf and cdf are given by:

$$
\begin{aligned}
& f_{\gamma}(x)=\frac{1}{\Gamma(m)}\left(\frac{m}{\bar{\gamma}}\right)^{m} x^{m-1} \exp \left(\frac{-m x}{\bar{\gamma}}\right), x \geq 0 \\
& F_{\gamma}(x)=1-\frac{1}{\Gamma(m)} \Gamma\left(m, \frac{m x}{\bar{\gamma}}\right)
\end{aligned}
$$

where $\Gamma(a, x)=\int_{x}^{\infty} \exp (-t) t^{a-1} d t$ denotes the complementary incomplete Gamma function and $m \geq 0.5$ is the fading severity index. If the fading index $m$ assumes a positive integer value, this may be simplified as

$$
F_{\gamma}(x)=1-\exp \left(-\frac{m x}{\bar{\gamma}}\right) \sum_{k=0}^{m-1} \frac{[m x / \bar{\gamma})]^{k}}{k !}
$$

\section{Nakagami-q Fading}

Fading signal amplitudes in satellite links that are subject to strong ionospheric scintillation tend to follow Nakagami-q distribution (also known as Hoyt distribution). In this case, the 
pdf of instantaneous branch SNR and its cdf are given by:

$$
\begin{aligned}
& f_{\gamma}(x)=\frac{1}{\bar{\gamma} \sqrt{1-b^{2}}} \exp \left[\frac{-x}{\left(1-b^{2}\right) \bar{\gamma}}\right] I_{0}\left[\frac{b x}{\left(1-b^{2}\right) \bar{\gamma}}\right], x \geq 0 \\
& F_{\gamma}(x)=I_{e}\left[b, x\left(1-b^{2}\right) \bar{\gamma}\right]
\end{aligned}
$$

where $-1 \geq b=\left(1-q^{2}\right) /\left(1+q^{2}\right) \leq 1,0 \leq q_{k} \leq \infty$, is the fading parameter and Rice's $I_{e}$-function is related to the first-order Marcum Q-function as

$$
\begin{aligned}
I_{e}(V / U, U) & =\frac{U}{W}[Q(\sqrt{U+W}, \sqrt{U-W}) \\
& -Q(\sqrt{U-W}, \sqrt{U+W})]
\end{aligned}
$$

while $W=\sqrt{U^{2}-V^{2}}$. The above model also includes the one-sided Gaussian $(b=1)$ and Rayleigh $(b=0)$ distributions as special instances.

\section{E. Weibull fading}

While this distribution is not widely used as others, recent experimental results have confirmed its validity for certain environments. For example, [17] presents a statistical analysis of data obtained by measuring narrow-band path loss at DECT (digital enhanced cordless telecommunications) frequency $(1.89 \mathrm{GHz})$ in an indoor environment. The best fitting distribution to the data is Weibull. The pdf and cdf of SNR which is Weibull distributed are given by

$$
\begin{aligned}
& f_{\gamma}(x)=\frac{m x^{m-1}}{\beta^{m}} \exp \left[-\left(\frac{x}{\beta}\right)^{m}\right], x \geq 0 \\
& F_{\gamma}(x)=1-\exp \left[-\left(\frac{x}{\beta}\right)^{m}\right]
\end{aligned}
$$

where $\beta=\bar{\gamma} / \Gamma(1+1 / m)$ and $m$ is the fading index or the shape parameter. For $m=1$, this reduces to the Rayleigh distribution for the fading amplitude.

\section{BOUNDS ON THE OUTAGE FOR GSC IN ARBITRARY FADING CHANNELS}

\section{A. Some basic results}

Here we list some basic results regarding order statistics without proofs. The interested reader is referred to [18]. Let $X_{(1)} \leq X_{(2)} \leq \ldots \leq X_{(n)}$ be the order statistics obtained from a random iid sample of size $n$ with pdf $f(x)$ and cdf $F(x)$. Then the density function of $X_{(r)}$ is given by [18]

$$
f_{X_{(r)}}(x)=\frac{n !}{(r-1) !(n-r) !}[F(x)]^{r-1}[1-F(x)]^{n-r} f(x)
$$

for $1 \leq r \leq n$. The joint pdf of $X_{(r)}$ and $X_{(s)}(1 \leq r<s \leq$ $n)$ is given by [18]

$$
\begin{aligned}
f_{X_{(r)}, X_{(s)}}(x, y) & =\frac{n !}{(r-1) !(s-r-1) !(n-s) !} \\
& \times[F(x)]^{r-1}[F(y)-F(x)]^{s-r-1} \\
& \times[1-F(y)]^{n-s} f(x) f(y)
\end{aligned}
$$

for $-\infty \leq x<y<\infty$.

\section{B. Simple Bounds for the outage}

Suppose $\gamma_{(1)} \leq \gamma_{(2)} \leq \ldots \gamma_{(L)}$ represent the order statistics obtained by arranging the instantaneous SNRs $\gamma_{1}, \gamma_{2}, \ldots, \gamma_{L}$ in increasing order of magnitude. As GSC collects the best $M$ paths and combines them coherently, the GSC output SNR is

$$
\gamma_{\mathrm{g} s c}=\sum_{k=L-M+1}^{L} \gamma_{(k)} \text {. }
$$

Outage is the probability that the output SNR falls below a given threshold that depends on the modulation type and other system design requirements. The outage may be defined as

$$
P_{\text {out }}=F \gamma_{\mathrm{g} s c}\left(\gamma_{\mathrm{t} h}\right)=\operatorname{Pr}\left(\gamma_{\mathrm{g} s c} \leq \gamma_{\mathrm{t} h}\right) .
$$

As mentioned before, this outage is fairly difficult to compute in some cases and analytical expressions exist only for limited cases. Furthermore, we would like to bound the outage and error rates without using the mgf of the GSC output. For this reason, we adapt a result by Slimane [19], who develops bounds for a sum of independent lognormal random variables by using the cdf of the maximum.

In order to derive the outage bounds that do not require mgf, we note that

$$
\gamma_{(L)} \leq \gamma_{\mathrm{g} s c} \leq M \gamma_{(L)}
$$

which results in

$$
\operatorname{Pr}\left(M \gamma_{(L)}<\gamma_{\mathrm{t} h}\right) \leq \operatorname{Pr}\left(\gamma_{\mathrm{g} s c} \leq \gamma_{\mathrm{t} h}\right) \leq \operatorname{Pr}\left(\gamma_{(L)}<\gamma_{\mathrm{th}}\right) .
$$

To evaluate these upper and lower bounds, we need the distribution of $\gamma_{(L)}$, which is well known. For the iid case, the cdf of the maximum is readily obtained from (8) as $F_{\gamma_{(L)}}(x)=\left[F_{\gamma}(x)\right]^{L}$. Thus, these bounds can be evaluated as

$$
\left[F\left(\gamma_{\mathrm{th}} / M\right)\right]^{L} \leq P_{\text {out }} \leq\left[F\left(\gamma_{\mathrm{th}}\right)\right]^{L} .
$$

This is exact if $M=1$ and the bounds should be tight where $M \ll L$ (since the lower bound tends to be quite close to the "exact" curve). Note that these bounds can be applied to any fading model such as Rician, Nakagami, Weibull and others. When $M$ is not very small in comparison to $L$, then the above upper bound can be loose. This may improved by noting that

$$
M \gamma_{(1)} \leq \gamma_{\mathrm{g} s c} \leq M \gamma_{(L)}
$$

This leads to the following bounds:

$$
\operatorname{Pr}\left(M \gamma_{(L)}<\gamma_{\mathrm{th}}\right) \leq \operatorname{Pr}\left(\gamma_{\mathrm{g} s c} \leq \gamma_{\mathrm{t} h}\right) \leq \operatorname{Pr}\left(M \gamma_{(1)}<\gamma_{\mathrm{th}}\right)
$$

To evaluate these upper bound, we need the distribution of $\gamma_{(1)}$, which is well known. For the iid case, the cdf of the minimum is readily obtained from (8). Thus, we have

$$
\left[F\left(\gamma_{\mathrm{th}} / M\right)\right]^{L} \leq P_{\text {out }} \leq 1-\left[1-F\left(\gamma_{\mathrm{th}} / M\right)\right]^{L} .
$$

For the case of non identical fading, the bounds (14) and (17) can be readily generalized as

$$
\prod_{i=1}^{L} F_{i}\left(\gamma_{\mathrm{t} h} / M\right) \leq P_{\mathrm{out}} \leq \prod_{i=1}^{L} F_{i}\left(\gamma_{\mathrm{t} h}\right) \quad \text { and }
$$




$$
\prod_{i=1}^{L} F_{i}\left(\gamma_{\mathrm{th}} / M\right) \leq P_{\mathrm{out}} \leq 1-\prod_{i=1}^{L}\left[1-F_{i}\left(\gamma_{\mathrm{th}} / M\right)\right]
$$

where $F_{i}()$ denotes the cdf of the $i$-th branch.

\section{IMPROVED BOUNDS FOR OUTAGE}

The above bounds are simple, but may not be tight enough for some purposes. We note that

$$
\gamma_{(L)}+\gamma_{(L-1)} \leq \gamma_{\mathrm{g} s c} \leq \gamma_{(L)}+(M-1) \gamma_{(L-1)} \text { for } M \geq 2 .
$$

This leads to

$$
I\left(M-1, \gamma_{\mathrm{th}}\right) \leq \operatorname{Pr}\left(\gamma_{\mathrm{g} s c} \leq \gamma_{\mathrm{th}}\right) \leq I\left(1, \gamma_{\mathrm{th}}\right) .
$$

where both the lower and upper bounds are special cases of $I(\alpha, \epsilon)=\operatorname{Pr}\left(\gamma_{(L)}+\alpha \gamma_{(L-1)} \leq \epsilon\right)$. We next derive $I(\alpha, \epsilon)$ as a single integral of the pdf and cdf of the fading model for iid fading. For this purpose, we need the joint pdf of $\gamma_{(L-1)}$ and $\gamma_{(L)}$. This can be obtained from (9). We thus have

$$
\begin{aligned}
I(\alpha, \epsilon)= & \int_{0}^{\epsilon /(1+\alpha)} \int_{x}^{\epsilon-\alpha x} f(x, y) d y d x \\
= & \frac{L !}{(L-2) !} \int_{0}^{\epsilon /(1+\alpha)}\left[F_{\gamma}(x)\right]^{L-2} \\
& \times\left\{F_{\gamma}(\epsilon-\alpha x)-F_{\gamma}(x)\right\} f_{\gamma}(x) d x .
\end{aligned}
$$

Hence, (21) can be evaluated in terms of a single integral. For some fading models, $I(\alpha, \epsilon)$ can be derived in closedform. However, this can also computed readily for any fading model via numerical integration. These tighter bounds can be useful for many performance studies.

An alternative, interesting proof of (22) is also possible. This proof uses a certain Markov property of order statistics. It is well known that $\gamma_{(L)}$ conditioned on $\gamma_{(L-1)}=x$ is a random variable with cdf

$$
F(y)=\frac{F_{\gamma}(y)-F_{\gamma}(x)}{1-F_{\gamma}(x)} \quad \text { for } \quad y \geq x .
$$

As a result, the quantity $I(\alpha, \epsilon)$ can be expressed as the expectation of a conditional cdf: ie,

$$
I(\alpha, \epsilon)=E\left\{\frac{F_{\gamma}(\epsilon-\alpha x)-F_{\gamma}(x)}{1-F_{\gamma}(x)}\right\}
$$

where $E()$ denotes the average over the pdf of $x$. Using (8) with $n=r+1=L$, we find that $x$ is distributed with the pdf

$$
\frac{L !}{(L-2) !}[F(x)]^{L-2}\left[1-F_{\gamma}(x)\right] f_{\gamma}(x) .
$$

Therefore, we readily obtain (22).

We now illustrate the closed-form evaluation of (22) for iid Rayleigh fading. Using (1), we can readily show that

$$
\begin{aligned}
I(\alpha, \epsilon \bar{\gamma}) & =c \int_{0}^{\epsilon /(1+\alpha)}\left(1-e^{-x}\right)^{L-2}\left\{e^{-x}-e^{-[\epsilon-\alpha x]}\right\} e^{-x} d x \\
& =c \sum_{r=0}^{L-2}(-1)^{r}\left(\begin{array}{c}
L-2 \\
r
\end{array}\right)\left[\left(\frac{1-e^{-(r+2) \epsilon /(1+\alpha)}}{r+2}\right)\right. \\
& \left.-e^{-\epsilon}\left(\frac{1-e^{-(r+1-\alpha) \epsilon /(1+\alpha)}}{r+1-\alpha}\right)\right]
\end{aligned}
$$

where $c=L ! /(L-2) !$.

We note that (22) can readily be generalized for ind fading environments. We can show that

$$
\begin{aligned}
J(\alpha, \epsilon)= & \operatorname{Pr}\left(\gamma_{(L)}+\alpha \gamma_{(L-1)} \leq \epsilon\right) \\
= & \int_{0}^{\epsilon /(1+\alpha)} \int_{x}^{\epsilon-\alpha x} f(x, y) d y d x \\
= & \sum_{r=1}^{L} \sum_{s=1}^{L} \int_{0}^{\epsilon /(1+\alpha)} \prod_{t=1, t \neq r, s}^{L} F_{\gamma, t}(x) \\
& \times\left\{F_{\gamma, r}(\epsilon-\alpha x)-F_{\gamma, r}(x)\right\} f_{\gamma, s}(x) d x .
\end{aligned}
$$

For the iid case, $F_{\gamma, *}(x)=F_{\gamma}(x)$ and this collapses to (22). This leads to

$$
J\left(M-1, \gamma_{\mathrm{th}}\right) \leq \operatorname{Pr}\left(\gamma_{\mathrm{g} s c} \leq \gamma_{\mathrm{t} h}\right) \leq J\left(1, \gamma_{\mathrm{t} h}\right) .
$$

\section{ERROR RATE BOUNDS FROM THE OUTAGE BOUNDS}

The average error rate is obtained by integrating the conditional error probability, $P_{e}(\gamma)$ over the pdf of $\gamma_{\mathrm{g} s c}$. We however express the average error probability in terms of the cdf of $\gamma_{\mathrm{g} s c}$. This can readily be done as

$$
\begin{aligned}
\bar{P}_{e} & =\int_{0}^{\infty} P_{e}(\gamma) f_{\gamma_{\mathrm{g} s c}}(\gamma) d \gamma \\
& =\int_{0}^{\infty}-P_{e}^{\prime}(\gamma) F_{\gamma_{\mathrm{g} s c}}(\gamma) d \gamma
\end{aligned}
$$

where $P_{e}^{\prime}(\gamma)$ denotes the derivative of the conditional error rate. Eq. (29) is obtained by integration by parts method. Combining this with (14), we find

$\int_{0}^{\infty}-P_{e}^{\prime}(\gamma)\left[F\left(\frac{\gamma}{M}\right)\right]^{L} d \gamma \leq \bar{P}_{e} \leq \int_{0}^{\infty}-P_{e}^{\prime}(\gamma)[F(\gamma)]^{L} d \gamma$

This can now be applied to any modulation format and any fading model (Section 2). Several derivatives of the conditional error probability expressions are shown in Table 1.

For example, let us illustrate the use of (30) for bounding the error rates of NCFSK and DPSK in Rayleigh fading. Using the corresponding derivative in Table 1 and the cdf (1), we have

$$
\begin{aligned}
I_{1}(\alpha) & =\frac{a}{2} \int_{0}^{\infty}\left(1-e^{-\alpha x / \bar{\gamma}}\right)^{L} e^{-a x} d x \\
& =\frac{a}{2} \sum_{r=0}^{L}(-1)^{r}\left(\begin{array}{l}
L \\
r
\end{array}\right)\left(\frac{1}{a+\alpha r / \bar{\gamma}}\right) .
\end{aligned}
$$

Thus the average error rates for the two modulation techniques can be bounded as

$$
I_{1}\left(\frac{1}{M}\right) \leq \bar{P}_{e} \leq I_{1}(1) .
$$

We next illustrate the use of (30) for bounding the error rates of BPSK and CFSK in Rayleigh fading.

$$
\begin{aligned}
I_{2}(\alpha) & =\frac{1}{2} \sqrt{\frac{a}{\pi}} \int_{0}^{\infty}\left(1-e^{-\alpha x / \bar{\gamma}}\right)^{L} e^{-a x} x^{-\frac{1}{2}} d x \\
& =\frac{\sqrt{a}}{2} \sum_{r=0}^{L}(-1)^{r}\left(\begin{array}{l}
L \\
r
\end{array}\right)\left(\frac{1}{\sqrt{a+\alpha r / \bar{\gamma}}}\right) .
\end{aligned}
$$


Thus the error rate can be bounded as

$$
I_{2}\left(\frac{1}{M}\right) \leq \bar{P}_{e} \leq I_{2}(1) .
$$

Similarly for QPSK, MSK and $M_{c}$-QAM, we can show that if

$$
\begin{aligned}
I_{3}(\alpha) & =\sum_{r=0}^{L}(-1)^{r}\left(\begin{array}{l}
L \\
r
\end{array}\right)\left\{2 q \sqrt{a}\left(\frac{1}{\sqrt{a+\alpha r / \bar{\gamma}}}\right)\right. \\
& \left.-\frac{q^{2}}{\sqrt{\pi(a+\alpha r / \bar{\gamma})}} \sin ^{-1}\left[\frac{1}{\sqrt{1+\frac{a}{(a+\alpha r / \bar{\gamma})}}}\right]\right\},
\end{aligned}
$$

then the average error rates for the two modulation techniques are bounded as

$$
I_{3}\left(\frac{1}{M}\right) \leq \bar{P}_{e} \leq I_{3}(1) .
$$

Several other error bounds can also be derived. These will be reported in a forthcoming journal paper.

The above can indeed be improved by combining (22) and (29). Thus an improved set of bounds are derived as

$$
\int_{0}^{\infty}-P_{e}^{\prime}(\gamma) I(M-1, \gamma) d \gamma \leq \bar{P}_{e} \leq \int_{0}^{\infty}-P_{e}^{\prime}(\gamma) I(1, \gamma) d \gamma
$$

Details are omitted for brevity.

\section{TABLE I}

CEP DERIVATIVE FOR SEVERAL COMMON MODULATION SCHEMES.

\begin{tabular}{|l|l|}
\hline $\begin{array}{l}\text { Modulation/ } \\
\text { Detection }\end{array}$ & $\begin{array}{l}\text { Negative derivative } \\
-P_{e}(\gamma)\end{array}$ \\
\hline $\begin{array}{l}\text { BPSK }(a=1) \\
\text { CFSK }\left(a=\frac{1}{2}\right)\end{array}$ & $0.5 \frac{e^{-a \gamma} a}{\sqrt{\pi} \sqrt{a \gamma}}$ \\
\hline $\begin{array}{l}\text { NCFSK }\left(a=\frac{1}{2}\right) \\
\text { DPSK }(a=1)\end{array}$ & $\frac{1}{2} e^{-a \gamma} a$ \\
\hline $\begin{array}{l}\text { QPSK }\left(q=a=\frac{1}{2}\right) \\
\text { MSK }\left(q=a=\frac{1}{2}\right) \\
M_{c} \text {-QAM }^{a}\left(q=1-\frac{1}{\sqrt{M_{c}}}\right)\end{array}$ & $2 \frac{q e^{-a \gamma} a}{\sqrt{\pi} \sqrt{a \gamma}}-2 \frac{q^{2} \operatorname{erfc}(\sqrt{a \gamma}) e^{-a \gamma} a}{\sqrt{\pi} \sqrt{a \gamma}}$ \\
\hline $\operatorname{CDE}^{-B P S K}{ }^{b}$ & $\frac{e^{-\gamma}}{\sqrt{\pi} \sqrt{\gamma}}-1.0 \frac{\operatorname{erfc}(\sqrt{\gamma}) e^{-\gamma}}{\sqrt{\pi} \sqrt{\gamma}}$ \\
\hline MDPSK & $\frac{1}{\pi} \int_{0}^{\pi-\pi / M} g(\theta) \exp [-\gamma g(\theta)] d \theta^{c}$ \\
\hline MPSK & $\frac{1}{\pi} \int_{0}^{\pi-\pi / M} h(\theta) \exp [-\gamma h(\theta)] d \theta^{d}$ \\
\hline
\end{tabular}

${ }^{a} a=3 / 2 /\left(M_{c}-1\right)$.

${ }^{b}$ Coherent detection of differentially encoded BPSK.

${ }^{c} g(\theta)=\frac{\sin ^{2}(\pi / M)}{1+\cos (\pi / M) \cos \theta}$

${ }^{d} h(\theta)=\frac{\sin ^{2}(\pi / M)}{\sin ^{2} \theta}$.

\section{NumericAl RESUlts AND CONCLUSION}

Fig. 1 compares the tightness of the upper and lower bounds (both simple and improved) as well as exact outage probability for $\operatorname{GSC}(2,8)$ and $\operatorname{GSC}(5,8)$ in iid Rayleigh fading. Note that for $\mathrm{M}=2$, the improved upper \& lower bounds coincide with the curve corresponding to the exact outage rate of error

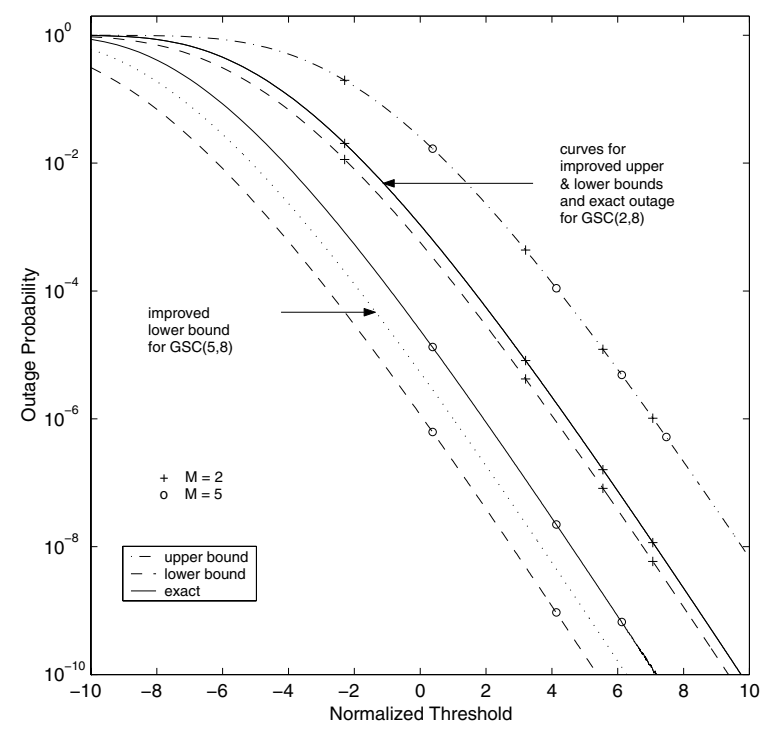

Fig. 1. Outage Probability for $\operatorname{GSC}(2,8)$ and $\operatorname{GSC}(5,8)$ in iid Rayleigh fading.

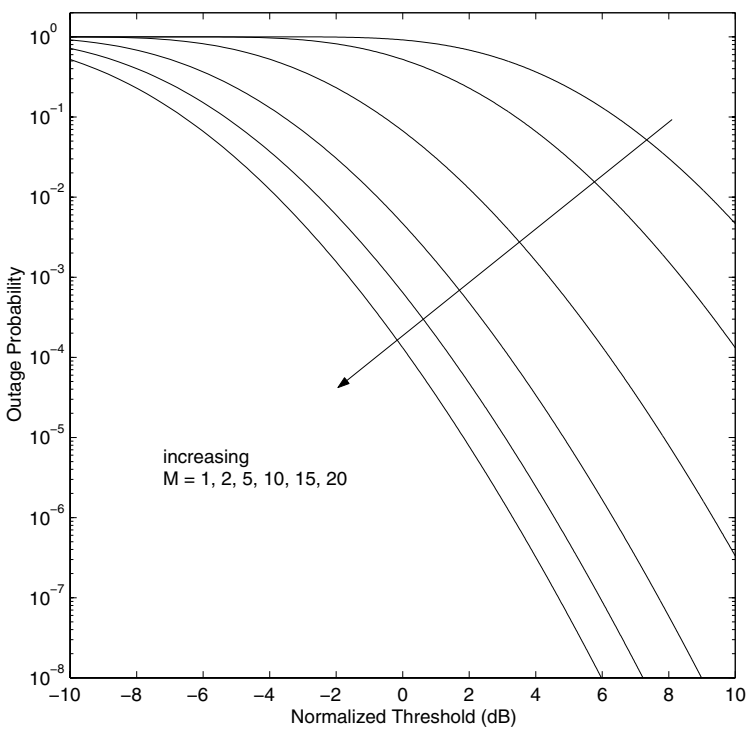

Fig. 2. Simple outage probability bounds for $\operatorname{GSC}(M, 30)$ in mixed fading.

probability of $\operatorname{GSC}(2,8)$ receiver. For $\mathrm{M}=5$, the improved upper bound coincides with the exact performance of $\operatorname{GSC}(2,8)$. It is apparent that the lower bounds are much tighter than the upper bounds particularly when $M \ll L$.

Fig. 2 is the outage probability in a mixed fading environment plotted as a function of normalized threshold (in $\mathrm{dB}$ ). The total number of multipaths is 30 . GSC performance when $\mathrm{M}=1,2,5,10,15$ and 20. We assume an exponential multipath intensity profile (MIP) with a decay factor $\delta=0.7$. The first 15 multipaths are Rician faded ( $K=3$ ), the next five multipaths are Rayleigh faded $(m=1)$ and the remaining 10 multipaths are Nakagami-m faded $(m=0.75)$.

Fig. 3 plots the ABER as a function of average SNR per bit for BPSK with GSC in ind Nakagami-m fading $(m=0.75)$ and $L=15$. The exponential MIP decay factor $\delta=0.7$. 


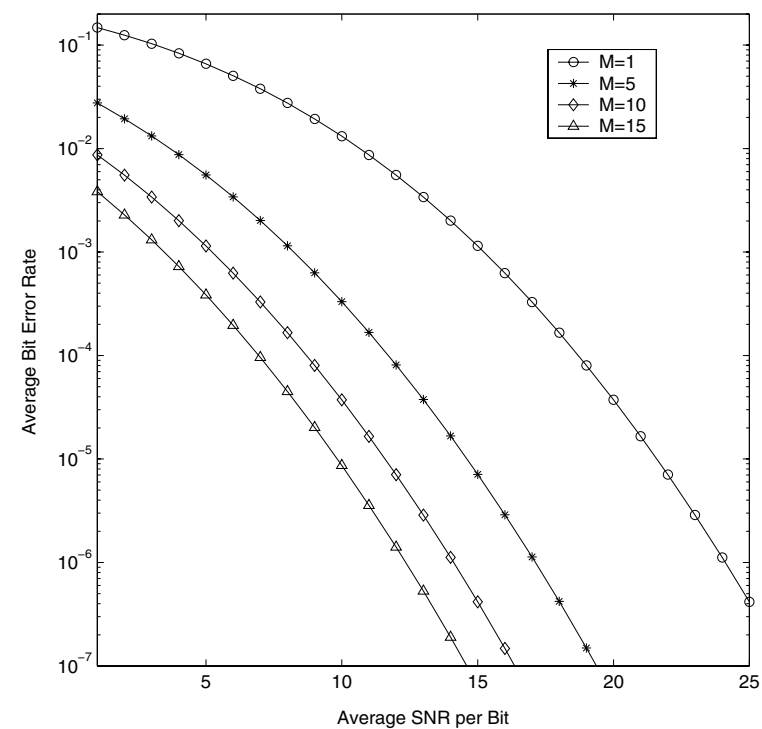

Fig. 3. Simple bounds for the average bit error rate of BPSK with GSC(M,15) receiver.

The mgf of a sum of ordered independent random variables is needed for computing the outage probability and error rates of a generalized selection combiner. Unfortunately, this mgf is either not known for generalized fading channels or does not exist in simple forms. This paper has derived new upper and lower outage bounds for the GSC over generalized fading channels. These bounds were further used to derive new bounds for the bit error rate and symbol error rates. Using these bounds, one can compute outage probability and average error rates for $\operatorname{GSC}(M, L)$ in myriad of fading environments and modulation techniques.

\section{REFERENCES}

[1] Ning Kong and L.B. Milstein, "SNR of generalized diversity selection combining with nonidentical Rayleigh fading statistics," IEEE Trans. Commun., vol. 48, pp. 1266 - 1271, Aug. 2000.

[2] N. Kong and L. Milstein, "Average SNR of a generalized diversity selection combining scheme," IEEE Commun. Lett., vol. 3, pp. 57-59, Mar. 1999.

[3] N. Kong, L. B. Milstein, "Average SNR of a generalized diversity selection combining scheme," in IEEE ICC, pp. 1556-1558, IEEE, June 1998.

[4] T. Eng, N. Kong, and L. B. Milstein, "Comparison of diversity combining techniques for Rayleigh-fading channels," IEEE Trans. Commun., pp. 1117-1129, Sept. 1996.

[5] N. Kong, T. Eng, and L. B. Milstein, "A selection combining scheme for rake receivers," in IEEE ICUPC, pp. 426-430, IEEE, 1995.

[6] A. Annamalai and C. Tellambura, "Error rates for Nakagami-m fading multichannel reception of binary and M-ary signals," IEEE Trans. Commun., vol. 49, pp. 58-68, Jan. 2001.

[7] A. Annamalai and C. Tellambura, "Error rates of hybrid SC/MRC diversity systems on Nakagami-m channels," in Wireless Communications and Netwroking conference, pp. 227-231, IEEE, Sept. 2000.

[8] M. Alouini and M. Simon, "Performance of coherent receivers with hybrid SC/MRC over nakagami-m fading channels," IEEE Trans. Veh. Technol., vol. 48, pp. 1155-1164, July 1999.

[9] R. Wong, A. Annamalai, and V. K. Bhargava, "Evaluation of predetection diversity techniques for rake receivers," in IEEE PACRIM'97, pp. 227-230, IEEE, Aug. 1997.

[10] M.-S. Alouini, M. K. Simon, "An MGF-based performance analysis of generalized selection combining over Rayleigh fading channels," IEEE Trans. Commun., vol. 48, pp. 401-415, Mar. 2000.
[11] Y. Ma and C. C. Chai, "Unified error probability analysis for generalized selection combining in Nakagami fading channels," IEEE J. Select. Areas. Commun., vol. 18, pp. $2198-2210$, Nov. 2000.

[12] A. Annamalai and C. Tellambura, "A new approach to performance evaluation of generalized selection diversity receivers in wireless channels," in IEEE Vehicular Technology Conference, pp. 2404-2408, IEEE, May 2001.

[13] A. Annamalai, G. Deora and C. Tellambura, "Unified error probability analysis for generalized selection diversity in Rician fading channels," in IEEE Vehicular Technology Conference, pp. 2042-2046, IEEE, 2002.

[14] H. Suzuki, "A statistical model for urban radio propagation," IEEE Trans. Veh. Technol., vol. 27, pp. 673-680, July 1977.

[15] W. R. Braun and U. Dersch, "A physical mobile radio channel model," IEEE Trans. Veh. Technol., vol. 40, pp. 472-482, Feb. 1991.

[16] M. Nakagami, "The m-distribution, a general formula of intensity distribution of rapid fading," in Statistical Methods in Radio Wave Propagation (W. G. Hoffman, ed.), Oxford, England: Pergamon, 1960.

[17] F. Babich and G. Lombardi, "Statistical analysis and characterization of the indoor propagation channel," IEEE Trans. Commun., vol. 48, pp. 455-464, Mar. 2000.

[18] H. A. David, Order Statistics. New York: John Wiley \& Sons, 2nd ed., 1981.

[19] S. Ben Slimane, "Bounds on the distribution of a sum of independent lognormal random variables," IEEE Trans. Commun., vol. 49, pp. 975978, June 2001. 